Meta

Journal des traducteurs

Translators' Journal

\title{
Néologismes et contact des langues
}

\section{Ambra Caputo, Emanuela Enrico et Fabienne Masucci}

Volume 32, numéro 3, septembre 1987

La fertilisation terminologique dans les langues romanes

URI : https://id.erudit.org/iderudit/002202ar

DOI : https://doi.org/10.7202/002202ar

Aller au sommaire du numéro

Éditeur(s)

Les Presses de l'Université de Montréal

ISSN

0026-0452 (imprimé)

1492-1421 (numérique)

Découvrir la revue

Citer cet article

Caputo, A., Enrico, E. \& Masucci, F. (1987). Néologismes et contact des langues. Meta, 32(3), 267-272. https://doi.org/10.7202/002202ar d'utilisation que vous pouvez consulter en ligne.

https://apropos.erudit.org/fr/usagers/politique-dutilisation/ 


\title{
NÉOLOGISMES ET CONTACT DES LANGUES
}

\author{
Ambra Caputo, Emanuela ENRICo et FabienNe MasucCi \\ Université de Rome, Rome, Italie
}

La problématique que nous aborderons aujourd'hui s'insère dans le cadre plus vaste d'une étude diachronique et synchronique sur la perméabilité de la langue française et de la langue italienne à l'emprunt à l'anglo-américain.

Aujourd'hui, nous présenterons les résultats partiels d'une enquête que nous avons effectuée dans la presse hebdomadaire d'information et d'opinion, pour les années 1981, 1983, 1985, à savoir l'Express, le Nouvel Observateur, et le Point et, parallèlement, l'Espresso, Panorama et l'Europeo. Notre corpus se compose actuellement de 4300 items pour le français et de $\mathbf{4 8 0}$ pour la langue italienne, notre étude n'étant pas encore informatisée, il s'agit des résultats partiels de notre enquête.

Ces items se distribuent de la façon suivante :

CORPUS FRANCAIS

Nombre total d'items : 4300

Les emprunts ${ }^{1}$ représentent dans le corpus français $\quad 83,25 \%$

Les calques $12,55 \%$

Les néologismes $\quad 4,20 \%$

Parties du discours dans lesquelles l'occurrence de l'emprunt est la plus forte :

Noms $=91,4 \%$ dont $22,5 \%$ ayant une marque d'intégration

Adjectifs $=4,4 \% \quad$ dont $18,7 \%$ ayant une marque d'intégration

Verbes $=3,9 \%$ dont $47,7 \%$ ayant une marque d'intégration

Pourcentage se référant au contexte sémantique :

Emprunts employés avec valeur dénotative $\quad 56,21 \%$

Emprunts employés avec valeur connotative $\quad 35,60 \%$

Emprunts employés avec guillemets/italiques $\quad 8,19 \%$

CORPUS ITALIEN

Nombre total d'items : 4880

Les emprunts représentent dans le corpus italien $\quad 82,7 \%$

Les calques $\quad 11,8 \%$

Les néologismes $\quad 5,5 \%$ 
Parties du discours dans lesquelles l'occurrence de l'emprunt est la plus forte :

Noms $=89,5 \%$

Adjectifs $=6,5 \%$

Verbes $=4 \% \quad$ dont $37,5 \%$ ayant une
Pourcentages se reférant au contexte sémantique : dont $24,5 \%$ ayant une marque d'intégration

dont $7,6 \%$ ayant une marque d'intégration

Emprunts employés avec valeur dénotative : $78 \%$

Emprunts employés avec valeur connotative : $\quad 29,4 \%$

Emprunts employés avec guillemets/italiques : $\quad 2,6 \%$

Notre analyse a été effectuée à l'aide de fiches que nous avons préalablement élaborées selon le schéma suivant :

\begin{tabular}{llll}
$1^{\text {re }}$ semaine & $2^{\mathrm{e}}$ semaine & $3^{\mathrm{e}}$ semaine & \\
\hline Express & Express & Express & JANVIER \\
\hline Le Point & Le Point & Le Point & FÉVRIER \\
\hline Nouvel & Nouvel & Nouvel & MARS \\
Observateur & Observateur & Observateur & \\
\hline
\end{tabular}

Cette méthode répondait à l'exigence d'analyser la fluctuation de l'emprunt dans une perspective également diachronique ; l'analyse visait essentiellement à : $a$ ) mesurer le nombre global des items relevés, $b$ ) analyser le rapport entre l'occurrence des items et le sujet traité dans l'article, $c$ ) analyser la qualité des items relevés par rapport au contexte d'intégration morphologique (suffixes, préfixes, etc.) et sémantique (emploi dénotatif et/ou connotatif, présence ou absence de guillemets et d'italiques dans l'article), $d$ ) analyser les modes de transposition propres à chacune des deux langues dans la perspective d'une analyse contrastive.

C'est, à présent, sur l'aspect néologique que nous allons porter notre attention. Toutefois, avant d'entrer dans les détails, il nous semble important de fournir quelques précisions d'ordre méthodologique. La première se réfêre au repérage du néologisme qui présente, notamment dans le cas de la néologie par contact des langues de nombreuses difficultés. Dans notre enquête nous nous sommes appuyées sur les critères de la datation, de la fréquence et de la modalité ${ }^{2}$, nous avons analysé les trois grandes catégories d'items (les emprunts, les calques et les néologismes) comme faisant partie du processus néologique en tant que stades différentiels du contact des langues. Dans cette perspective, l'emprunt représenterait le stade primaire. C'est précisément sur ce stade que nous avons orienté nos réflexions. Une des préoccupations majeures lors de notre enquête a été celle de définir la notion même de l'emprunt à l'anglo-américain. En ce qui concerne l'emprunt, la définition est loin d'être simple et elle est susceptible de plusieurs classifications en fonction de différents critères ; c'est ainsi, que nous nous sommes appuyées sur la théorie de Valentín García Yebra Teoría y prática de la traduccion (Gredos, 1982), qui semblait le mieux correspondre à notre enquête. Ici, nous n'avons retenu que les critères les plus pertinents en raison de la perspective adoptée :

a) les emprunts véhiculant un signifié qui dans un premier temps n'est pas attesté dans la langue cible et qui enrichirait celle-ci d'une notion ou d'un signifié que l'on ne saurait désigner autrement. Ces emprunts sembleraient traduire une sorte de " décalage " lexical, décalage déterminé par la pression néologique due au contact des langues.

b) Les emprunts véhiculant un signifié qui dans la langue cible pourrait tout aussi bien être exprimé par un signe préexistant. Dans ce cas, il s'agirait plutôt d'un choix sty- 
listique déterminé par des facteurs complexes parmi lesquels l'impact des modes culturelles et linguistiques semble prépondérant.

c) Les emprunts qui sur le plan sémantique diffèrent sensiblement d'une langue à l'autre, par exemple " jogging " qui en français correspond à " course " et à "survêtement " alors qu'en italien le même terme ne correspond qu'au signifié "course ".

Parallèlement à cette classification, nous avons porté notre attention sur certains aspects tels, la modalité de transposition des mots construits étrangers, le rapport entre qualité de l'item et sujet traité dans l'article et des facteurs comme la fluctuation de l'emprunt et la multiplication des occurrences dans le même article, facteurs qui nous semblent être étroitement liés au processus néologique.

En ce qui concerne les emprunts traduisant une sorte de décalage lexical, nous avons retenu, au cours de notre analyse, certains éléments qui nous ont semblé essentiels.

- En ce qui concerne l'ordre déterminé-déterminant, par exemple nous avons remarqué que la fluctuation peut jouer un rôle essentiel dans l'intégration de l'emprunt. Dans les fichiers de 1985 l'ordre " disques-vidéo " et " cassettes-vidéo " a complètement détrôné l'ordre " vidéo-disques " et " vidéo-cassettes " apparaissant dans le fichier se référant à 1981 .

- En ce qui concerne les modes de transposition, nous avons pu constater une régularité presque absolue de ce procédé et une correspondance dans la formation des affixes (due à l'origine commune de l'italien et du français). Quelques exemples relatifs aux calques avec suffixation illustreront mieux ces aspects :

-er qui devient eur/ore par ex. décideur/decisore

-ing qui devient age/aggio

-al qui devient al/ale

par ex. stockage/stoccaggio

par ex. managérial/manageriale

(Dans le cas de " managérial " la marque de complétion a suffi à franciser l'item.)

- En ce qui concerne la formation des verbes, nous avons remarqué la prédominance de la première conjugaison.

Ex. stocker - stoccare

flasher — flasciare

tester — testare

etc.

- Sur le plan du syntagme, nous avons pu constater une forte tendance à l'économie des prépositions.

Ex. plans (au pluriel) - épargne logement (au singulier)

il s'agit d'un exemple qui illustre aussi l'invariabilité du déterminant.

Ex. Service-recherches (au pluriel)

Casi-limite

Aree-sistema

Solutions-standards / Soluzioni-standard

Passons maintenant à l'emprunt déterminé par un choix stylistique. Avant d'aborder cet aspect, il nous semble indispensable de fournir quelques précisions.

Au cours de notre enquête, l'analyse du rapport entre sujet traité dans l'article et occurrence de l'emprunt ainsi que l'analyse du rapport entre occurrence de l'emprunt et présence de néologisme par contact a fait ressortir quelques éléments intéressants.

Dans les articles plus strictement scientifiques nous avons relevé deux tendances : 1) la fascination pour le terme américain et la difficulté objective que la traduction des termes implique, sclérosent ou tout du moins bloquent la créativité lexicale. 2) Lorsqu'un terme nouveau apparait il s'agit presque toujours d'un néologisme qui est 
déjà attesté dans la langue, l'auteur se limitant à fournir une sommaire tentative d'explication du signifié.

En revanche, le plus grand nombre d'emprunts apparaît dans les articles traitant de mode, tendances de la société, mœurs (en terme de pourcentage cela correspond à environ $70 \%$ du corpus analysé), toutefois il est d'autant plus vrai que c'est dans ce type de sujet que l'emprunt active un processus dynamique aboutissant à la création néologique, notamment lorsque, à l'intérieur du même article l'occurrence de l'emprunt est particulièrement forte. C'est précisément la multiplication des occurrences qui neutralise la motivation du choix stylistique, même dans le cas d'emprunts banalisés. C'est ce que nous avons pu remarquer au cours de notre analyse des fichiers de 1981/1983 pour le cas de "cool», détrôné par "décontract" et en italien de "look» détrôné par "imagine".

Nous nous sommes attachées à déterminer une typologie des stades du processus néologique que nos fichiers nous ont permis d'élaborer, et cela malgré la noninformatisation des données.

- SCHÉMA DU STADE STATIQUE

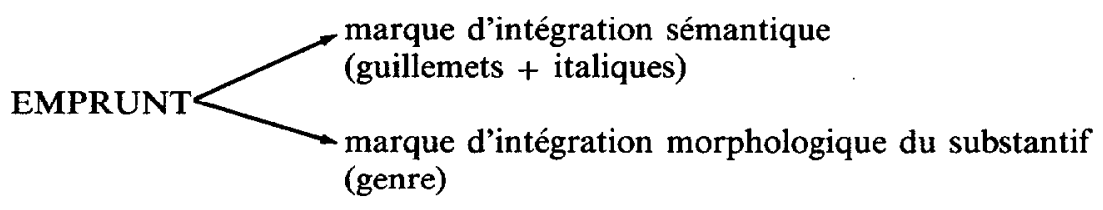

Ce schéma illustre les modifications que l'emprunt peut subir.

a) Il peut ou non se révéler avec une marque d'intégration sémantique, à savoir guillemets, italiques qui traduirait de la part du journaliste une sorte de malaise dans l'utilisation du néologisme lexical.

b) Les marques d'intégration morphologique se révèlent à deux niveaux différents : 1) détermination obligatoire du genre du substantif en français et en italien, révélé dans un premier temps par le choix de l'article. Ce choix semble être déterminé par les facteurs phonétiques propres au système phonétique de la langue cible et/ou, par l'influence d'une traduction potentielle. 2) Ce choix du genre de l'article sera confirmé ultérieurement par le morphème du genre qui sera suffixé au substantif emprunté.

SCHÉMA DU STADE DYNAMIQUE

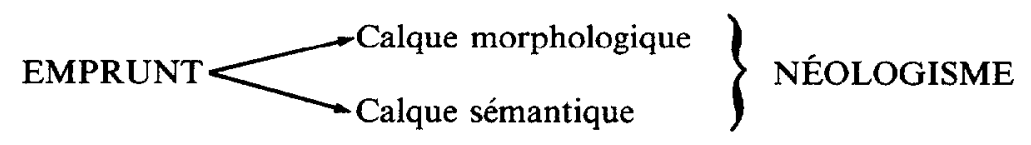

Le passage de l'emprunt au calque marque le passage du stade statique au stade dynamique. L'emprunt s'intègre dans la langue cible par le recours à des marques d'intégration morphologique, ou par le biais de la traduction. La création lexicale favorisée par le contact des langues se révèle être un facteur dynamique. D'autre part, si nous considérons comme vraie la notion de néologie ou plutôt de sa collocation entre la norme et l'usage, l'emprunt représenterait le premier stade de transgression, stade qui contient en soi la réintégration dans la norme. 
Ce stade illustre aussi le caractère flottant de la différence souvent établie entre calque et néologisme dans le domaine du contact des langues.

Avant d'aborder la partie illustrative, il nous semble essentiel de souligner l'importance de la multiplication de l'occurrence de l'emprunt favorisant le néologisme, ce qui a été maintes fois confirmé au cours de notre enquête. La multiplication des occurrences au sein d'un même article illustre l'itinéraire de l'emprunt favorisant la création lexicale. Le rapport est encore plus visible en ce qui concerne les articles se référant aux tendances de la société, là où les choix stylistiques viennent renforcer les facteurs dynamiques offerts par le contact des langues.

Autrement dit, dans la première partie de l'article, l'emprunt apparaît isolé, il prendra éventuellement, par la suite, des guillemets. Ces indices d'hésitation seront éliminés au fur et à mesure. C'est à ce moment que le néologisme fait son apparition, au début souvent entre guillemets, guillemets qu'il abandonne bientôt à mesure de son emploi ; le terme est alors intégré.

EXEMPLES ILLUSTRANT LA TYPOLOGIE DES STADES DU PROCESSUS NÉOLOGIQUE tirés de :

- Le Point, mars 1981

Stade statique

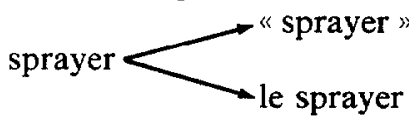

- Panorama, mars 1983

Stade statique

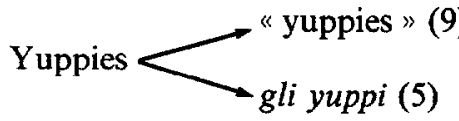

- L'Europeo, mars 1985

Stade statique

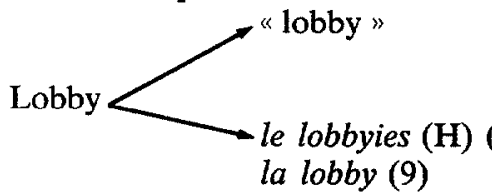

(12)
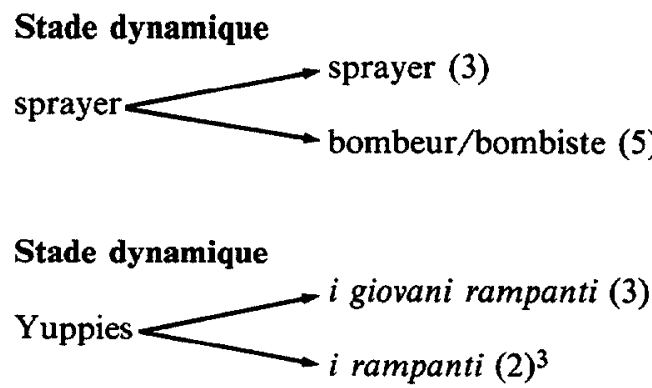

Stade dynamique

Stade dynamique

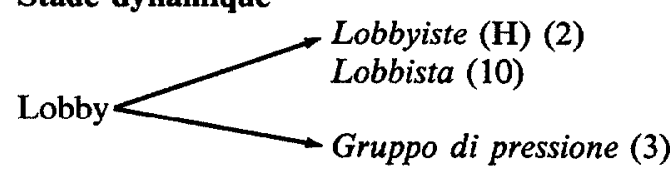

Légende : Chaque item est accompagné d'un numéro qui indique la fréquence de l'occurrence. Certains items portent une marque d'hypercorrection que nous avons notée $(\mathrm{H})$.

En conclusion, les facteurs statiques et les facteurs dynamiques semblent, au cours de notre enquête s'alterner. En effet, s'il est vrai que la presse écrite témoigne de plus en plus d'un processus plus général de stéréotypisation linguistique favorisée par la présence de certains éléments, tels l'économie linguistique renforcée par le modèle linguistique anglo-américain : substitution de l'ordre déterminé-déterminant, invariabilité du déterminant, économie des prépositions dans le syntagme, il est d'autant plus vrai que ce sont précisément ces éléments qui semblent préparer un vaste terrain d'accueil à l'emprunt. On pourrait donc avancer l'hypothèse que le contact linguistique offert par 
l'anglo-américain ne concernerait pas exclusivement l'emprunt, en tant qu'élément isolé, mais bien plus l'emprunt global à un modèle de discours : le modèle angloaméricain. L'emprunt lexical apparaîtrait donc comme l'une des manifestations d'un vaste processus qui touche aussi à la stratégie du discours et qui n'est pas sans effets sur certains aspects de la structure même de la langue.

Les deux tendances observées au cours de notre enquête sembleraient donc s'exclure mutuellement : l'une étant dynamique, l'autre étant statique. Autrement dit, l'emprunt serait d'une part le stade primaire, stade, nous disons, de transgression du processus néologique et contiendrait en soi les potentialités aptes à le réintégrer dans le système linguistique et à le réadapter dans la norme : d'autre part, l'emprunt freinerait la création lexicale et aboutirait au statisme. Nous ne pouvons pas apporter de réponses définitives à cette question, toutefois les résultats de notre enquête, quoique partiels et limités au domaine analysé, semblent globalement affirmer une certaine prépondérance de l'aspect dynamique offert par le contact des langues.

Notes

1. À Noter, que le pourcentage mentionné plus haut, et qui peut sembler extrêmement élevé est dû au fait que chaque occurrence a été comptabilisée. C'est un choix méthodologique qui a été motivé par la nécessité d'une analyse de la fluctuation de l'emprunt et de la multiplication des occurrences au cours des années analysées.

2. Cf. Riffeterre, Guilbert, Gardin, Lefêvre, Marcellesi, Mortureux.

3. Il est intéressant de noter que l'adjectif Rampanti qui signifie : qui grimpent, subit un glissement sémantique, car il s'enrichit d'une connotation nouvelle : monter dans l'échelle sociale. D'autre part, du calque sémantique $i$ giovani rampanti, littéralement, les jeunes grimpants, il devient "grimpant ". Le contact des langues a done déterminé le changement de catégorie grammaticale, dans le cas présent, de l'adjectif au substantif, et il a d'autre part, favorisé l'enrichissement sémantique. 